Supporting Information for

\title{
A Synthesis of Maternal Transfer of Mercury in Birds: Implications for Altered Toxicity Risk
}

Joshua T. Ackerman*,1, Mark P. Herzog ${ }^{1}$, David C. Evers ${ }^{2}$, Daniel A. Cristol ${ }^{3}$, Kevin P. Kenow ${ }^{4}$, Gary H. Heinz ${ }^{5}$, Raphael A. Lavoie ${ }^{6}$, Rebecka L. Brasso ${ }^{7}$, Mark L. Mallory ${ }^{8}$, Jennifer F. Provencher $^{8}$, Birgit M. Braune ${ }^{9}$, Angela Matz ${ }^{10}$, Joel A. Schmutz ${ }^{11}$, Collin A. Eagles-Smith ${ }^{12}$, Lucas J. Savoy ${ }^{2}$, Michael W. Meyer ${ }^{13}$, and C. Alex Hartman ${ }^{1}$

${ }^{1}$ U.S. Geological Survey, Western Ecological Research Center, Dixon Field Station, 800 Business Park Drive, Suite D, Dixon, California 95620, United States

${ }^{2}$ Biodiversity Research Institute, 276 Canco Road, Portland, Maine 04103, United States

${ }^{3}$ College of William and Mary, Biology Department, P.O. Box 8795, Williamsburg, Virginia 23187, United States

${ }^{4}$ U.S. Geological Survey, Upper Midwest Environmental Sciences Center, 2630 Fanta Reed Road, La Crosse, Wisconsin 54603, United States

${ }^{5}$ U.S. Geological Survey, Patuxent Wildlife Research Center, BARC-East, Building 308, 10300 Baltimore Avenue, Beltsville, Maryland 20705, United States

${ }^{6}$ Groupe de Recherche Interuniversitaire en Limnologie et environnement aquatique (GRIL), Département de Sciences Biologiques, Université de Montréal, Pavillon Marie-Victorin, CP6128, Succ. Centre-ville, Montréal, Québec H3C 3J7, Canada

${ }^{7}$ Weber State University, Department of Zoology, 1415 Edvalson Dr, Ogden, UT 84408

${ }^{8}$ Acadia University, Biology Department, 15 University Drive, Wolfville, Nova Scotia B4P 2R6, Canada

${ }^{9}$ Environment and Climate Change Canada, National Wildlife Research Centre, Carleton University, Raven Road, Ottawa, Ontario K1A 0H3, Canada

${ }^{10}$ U.S. Fish and Wildlife Service, 1011 E. Tudor Road, Anchorage, Alaska 99503, United States

${ }^{11}$ U.S. Geological Survey, Alaska Science Center, 4210 University Drive, Anchorage, Alaska 99508, United States

${ }^{12}$ U.S. Geological Survey, Forest and Rangeland Ecosystem Science Center, 3200 SW Jefferson Way, Corvallis, Oregon 97331, United States

${ }^{13}$ Wisconsin Department of Natural Resources, 107 Sutliff Avenue, Rhinelander, Wisconsin 54501, United States

\section{Corresponding Author}

*e-mail: jackerman@usgs.gov (J.T.A.).

Total pages (including cover): 22

Tables: 3

Figures: 0 


\section{MATERIALS AND METHODS \\ Data compilation}

For herring gulls, black-legged kittiwakes, common eiders, and great black-backed gulls sampled at Corossol Island, Quebec, Canada, THg concentrations were determined on a dry weight basis in red blood cells rather than in whole blood (Table S1). Therefore, we converted $\mathrm{THg}$ concentrations in red blood cells on a dry weight basis to $\mathrm{THg}$ concentrations in whole blood on a wet weight basis. We first transformed THg concentrations in red blood cells on a dry weight basis to a wet weight basis using individual-specific moisture content of red blood cells. We then estimated the THg concentration in the plasma portion of the whole blood using the equation: $\mathrm{THg}$ plasma $\mu \mathrm{g} / \mathrm{g} \mathrm{ww}=0.0119 \times \mathrm{THg}$ red blood cells $\mu \mathrm{g} / \mathrm{g} \mathrm{ww}+0.0019\left(R^{2}=0.60\right.$, $n=30$; Lavoie, unpublished). Finally, we estimated the THg concentration in whole blood by adding the $\mathrm{THg}$ concentrations in the red blood cell and plasma portions of whole blood by assuming that red blood cells accounted for $44.2 \%$ of the whole blood volume (which is a weighted average from the literature) using the equation: $\mathrm{THg}$ whole blood $\mu \mathrm{g} / \mathrm{g} \mathrm{ww}=(\mathrm{THg}$ red blood cells $\mu \mathrm{g} / \mathrm{g} \mathrm{ww} \times 0.442)+(\mathrm{THg}$ plasma $\mu \mathrm{g} / \mathrm{g} \mathrm{ww} \times[1-0.442])$.

\section{Mercury determination}

THg content was determined using several analytical approaches (Milestone DMA-80 Direct Mercury Analyzer, Milestone, Monroe, Connecticut, USA; Nippon MA-3000 Direct Mercury Analyzer, Nippon Instruments North America, College Station, Texas, USA; Nippon Instruments MA-2000 Mercury Analysis System, Nippon Instruments North America, College Station, Texas, USA; Nippon Instruments Mercury SP-3D Analyzer, Nippon Instruments, Osaka, Japan; AMA254 Advanced Mercury Analyzer, St. Joseph, Michigan, USA; PerkinElmer ELAN 6000/6100, Waltham, Massachusetts, USA) and methods are available in previously published studies. $^{1-8}$

\section{Statistical analysis}

We conducted most statistical analyses using JMP® (version 14.3.0; SAS Institute Inc.) statistical software. We used linear mixed-effect and fixed-effect models to examine the relationship between THg concentrations in mothers' blood and their eggs. For the mixed-effect models, the Satterthwaite method was used to estimate the denominator degrees of freedom. 
Using R (version 3.6.0; www.r-project.org) statistical software, we calculated the marginal $R^{2}$ values (hereafter $R_{m}^{2}$ ) to describe the proportion of variance explained by the fixed-effects, ${ }^{9,10}$ which, for models where there were no other fixed-effects in the models, specifically assessed the explanatory power of $\mathrm{THg}$ concentrations in blood for predicting $\mathrm{THg}$ concentrations in eggs (Table S2) and vice versa (Table S3). Conditional $R^{2}$ values (hereafter $R_{c}^{2}$ ) also were included in Tables S2 and S3 and describe the proportion of variance explained by both the fixed- and random-effects in the model. Egg and blood THg concentrations were $\log _{\mathrm{e}}$-transformed prior to analysis. In the figures, we present either the individual egg $\mathrm{THg}$ concentration (when only one egg was sampled from the clutch) or the geometric mean $\mathrm{THg}$ concentration and the range (minimum to maximum) of $\mathrm{THg}$ concentrations of all the eggs within the clutch (when the complete clutch was collected) versus the maternal blood $\mathrm{THg}$ concentration. Table S2 provides test statistics, predictive equations, and variance for the following egg to maternal blood models.

\section{Egg to maternal blood relationship: differences among studies within the same species}

The relationship between THg concentrations in eggs and maternal blood might differ among studies of the same species due to factors such as differences among sites ${ }^{11}$ or the timing of female blood sampling. ${ }^{3}$ Therefore, we first examined the potential differences in this relationship within the same species but among studies in five species that each had 2-5 unique datasets (collected by separate researchers or using different methods), including tree swallows (five datasets), common loons (two datasets), mallards (three datasets), Carolina wrens (two datasets), and black-legged kittiwakes (two datasets; Table 1). We conducted separate analyses for each of these five species where THg concentration in individual eggs was the dependent variable and $\mathrm{THg}$ concentration in maternal blood and study (dataset) were fixed effects, $\mathrm{THg}$ concentration in maternal blood $\times$ study was included as an interaction, and unique nest identification was included as a random effect. In this model we statistically nested individual eggs within the clutch it came from when $>1$ egg was collected from the same nest (complete clutches). If the interaction between maternal blood $\mathrm{THg}$ concentration and study was nonsignificant, we removed the interaction from the model structure and reran the analysis. After using this model structure to test whether the relationship differed among studies, we conducted separate models for each study to estimate the species- and study-specific equations to predict THg concentrations in eggs from maternal blood. 
Egg to maternal blood relationship: by species

Second, we examined whether the relationship between $\mathrm{THg}$ concentrations in eggs and maternal blood differed among species. THg concentration in individual eggs was the dependent variable and $\mathrm{THg}$ concentration in maternal blood and species were fixed effects, $\mathrm{THg}$ concentration in maternal blood $\times$ species was included as an interaction, and unique nest identification and study were included as random effects. Each individual egg was statistically nested within the clutch and study (when more than one study was conducted on the same species). If the interaction between maternal blood THg concentration and species was nonsignificant, we removed the interaction from the model structure and reran the analysis. We excluded four species with small sample sizes ( $n=1$ nest and $n \leq 4$ eggs each for eastern phoebe, northern cardinal, and red-winged blackbird; and $n=2$ nests and $n=2$ eggs for great black-backed gull). To determine specific differences among species within the same taxonomic order, we repeated the analysis within each of the four orders where multiple species were studied (Anseriformes, Charadriiformes, Passeriformes, and Gaviiformes). After using this model structure to test whether the slopes and intercepts differed among species overall and within each taxonomic order, we conducted separate models for each species to estimate the species-specific equations to predict $\mathrm{THg}$ concentrations in eggs from maternal blood. These models were similar to the global model, but did not include species or maternal blood $\times$ species interaction (because models were conducted separately for each species) and study was included as a random effect only for the five species where $>1$ study per species was conducted (tree swallow, common loon, mallard, Carolina wren, and black-legged kittiwake).

\section{Egg to maternal blood relationship: by taxonomic order}

Third, we examined whether the relationship between THg concentrations in eggs and maternal blood differed among taxonomic orders. THg concentration in individual eggs was the dependent variable and $\mathrm{THg}$ concentration in maternal blood and taxonomic order were fixed effects, $\mathrm{THg}$ concentration in maternal blood $\times$ order was included as an interaction, and unique nest identification, study, and species were included as random effects. Each individual egg was statistically nested within the clutch, study, and species it came from. If the interaction between maternal blood $\mathrm{THg}$ concentration and order was not significant, we removed the interaction 
from the model structure and reran the analysis. After using this model structure to test whether the slopes and intercepts differed among orders, we conducted separate models for each order (except Procellariiformes because $n=3$ eggs) to estimate the order-specific equations to predict $\mathrm{THg}$ concentrations in eggs from maternal blood. The structures of these models were similar to the global model, but did not include order or maternal blood $\times$ order interaction (because models were conducted separately for each order). These models also excluded species as a random effect from taxonomic orders without more than one species sampled (Coraciiformes) and excluded study as a random effect from orders without more than one study conducted within a species (Coraciiformes).

\section{Egg to maternal blood relationship: captive studies}

Fourth, we used data from only the captive bird studies to investigate if the relationship between THg concentrations in eggs and maternal blood differed among captive birds kept in controlled conditions. We included data from two captive dosing studies, one on mallard ${ }^{4}$ and one on zebra finch (Cristol). $\mathrm{THg}$ concentration in individual eggs was the dependent variable and $\mathrm{THg}$ concentration in maternal blood and species were fixed effects, $\mathrm{THg}$ concentration in maternal blood $\times$ species was included as an interaction, and unique nest identification was included as a random effect. If the interaction between maternal blood $\mathrm{THg}$ concentration and species was not significant, we removed the interaction from the model structure and reran the analysis.

\section{Egg to maternal blood relationship: all taxa}

Fifth, because of the utility for a more universal equation across species to predict $\mathrm{THg}$ concentrations in eggs from $\mathrm{THg}$ concentrations in female bird blood for any species, we also produced a general equation using a model where THg concentration in individual eggs was the dependent variable and $\mathrm{THg}$ concentration in maternal blood was a fixed effect and unique nest identification, study, and species were included as random effects.

Maternal blood to egg relationship: by species, order, and all taxa

Finally, because many investigators sample eggs and may want to predict maternal blood THg concentrations (rather than the above egg predictions using blood), we restructured the 
statistical models and conducted separate tests for each 1) species: where female blood THg concentration was the dependent variable and geometric mean egg THg concentration in a clutch was a fixed effect and study was a random effect; 2) order: where female blood THg concentration was the dependent variable and geometric mean egg $\mathrm{THg}$ concentration in a clutch was a fixed effect and species and study were random effects; and 3) all taxa: where female blood THg concentration was the dependent variable and geometric mean egg THg concentration in a clutch was a fixed effect and species and study were random effects. The statistics and equations for predicting maternal blood $\mathrm{THg}$ concentrations from egg $\mathrm{THg}$ concentrations are presented only in Table S3 and are used for the predictions in Table 3.

\section{RESULTS}

\section{Egg to maternal blood relationship: differences among studies within the same species}

First, we examined the potential differences within the same species but among studies in five species that each had 2-5 unique datasets (Table 1).

\section{Tree swallows}

We had five unique datasets for tree swallows where the geographic location or timing of female blood sampling differed among studies. THg concentrations in eggs were positively correlated with THg concentrations in the mother's blood in tree swallows $\left(F_{1,158.5}=1101.46\right.$, $p<0.0001)$, but there were significant effects of study $\left(F_{4,146.0}=26.69, p<0.0001\right)$ and blood $\mathrm{THg}$ concentration $\times$ study interaction $\left(F_{4,148.3}=14.15, p<0.0001\right.$; final model: $n=568$ eggs, $R_{m}^{2}=0.94$; Figure 1a). The significant blood THg concentration $\times$ study interaction and study effect indicated that the relationship between $\mathrm{THg}$ concentrations in eggs and maternal blood differed among the tree swallow studies. The most noteworthy difference among these studies was a shallower slope in the study by Evers (Eq. 5; green triangles) which predicted relatively lower egg THg concentrations at higher blood THg concentrations (Figure 1a). However, three of the five studies had similar slopes (Eqs. 1, 2, and 4). Additionally, the short time difference in sampling a female's blood during incubation had a small influence on the predicted egg $\mathrm{THg}$ concentrations in the two studies by Ackerman et al. ${ }^{3}$ Specifically, for any predicted egg THg concentration, females bled immediately after clutch completion (early incubation) had lower blood THg concentrations than females bled after 6-10 days in incubation (mid to late 
incubation), indicating that females acquired additional THg after egg laying during incubation. The specific equations among these five studies to predict $\mathrm{THg}$ concentrations in eggs from maternal blood were (Table S2):

(1) $\ln \left(\right.$ Egg THg $\left.\frac{\mu g}{g} f w w\right)=0.8091 \times \ln \left(\right.$ Female Tree Swallow Blood THg $\left.\frac{\mu g}{g} w w_{w}\right)-1.3740$ (females bled during early incubation, ${ }^{3} n=171$ eggs, $\left.R_{m}^{2}=0.80\right)$

(2) $\ln \left(\right.$ Egg THg $\left.g_{\frac{\mu g}{g} f w w}\right)=0.7892 \times \ln \left(\right.$ Female Tree Swallow Blood THg $\left.\frac{\mu g}{g} w w\right)-1.2195$ (females bled during late incubation, ${ }^{3} n=52$ eggs, $R_{m}^{2}=0.70$ )

(3) $\ln \left(\right.$ Egg THg $\left.\frac{\mu g}{g} f w w\right)=0.9598 \times \ln \left(\right.$ Female Tree Swallow Blood THg $\left.\frac{\mu \underline{g}}{g} w w_{w}\right)-1.3412$ (females bled at clutch completion, ${ }^{2} n=162$ eggs, $R_{m}^{2}=0.97$ )

(4) $\ln \left(\right.$ Egg THg $\left.\frac{\mu g}{g} f w w\right)=0.8568 \times \ln \left(\right.$ Female Tree Swallow Blood THg $\left.\frac{\mu g}{g}_{w w}\right)-0.9689$ (Cristol) (females bled throughout incubation, $n=137$ eggs, $R_{m}^{2}=0.97$ )

(5) $\ln \left(\right.$ Egg THg $\left.g_{\frac{\mu g}{g} f w w}\right)=0.4549 \times \ln \left(\right.$ Female Tree Swallow Blood THg $\left.\frac{\mu g}{g} w w_{w}\right)-1.8412$ (Evers)

(females bled throughout incubation and nestling rearing, $n=46$ eggs, $R_{m}^{2}=0.63$ )

\section{Common loons}

We had two unique datasets for common loons. After removing the non-significant blood THg concentration $\times$ study interaction term $\left(F_{1,80.6}=1.08, p=0.30\right), \mathrm{THg}$ concentrations in eggs were positively correlated with THg concentrations in the mother's blood in common loons $\left(F_{1,88.8}=82.67, p<0.0001\right)$ and there was no influence of study $\left(F_{1,83.1}=2.18, p=0.14\right.$; final model: $n=119, R_{m}^{2}=0.46$; Figure $1 \mathrm{~b}$ ). This indicates that the relationship of THg concentrations in eggs and blood did not statistically differ between studies because there was substantially more variation in the study by Evers (Figure 1b). The study-specific equations between $\mathrm{THg}$ concentrations in eggs and maternal blood were (Table S2): 
(6) $\ln \left(\right.$ Egg THg $\left.\frac{\mu g}{g} f w w\right)=0.9753 \times \ln \left(\right.$ Female Common Loon Blood THg $\left.g_{\frac{\mu g}{g} w w}\right)-0.4318$ (Wisconsin, ${ }^{7}$ $n=29$ eggs, $\left.R_{m}^{2}=0.91\right)$

(7) $\ln \left(\right.$ Egg THg $\left.\frac{\mu g}{g} f w w\right)=0.7116 \times \ln \left(\right.$ Female Common Loon Blood THg $\left.\frac{\mu g}{g} w w\right)-0.6937$ (Evers, northeastern USA, $n=90$ eggs, $R_{m}^{2}=0.42$ )

\section{Mallard}

We had three unique datasets for mallards, including one field study and two captive studies where the timing of female blood sampling differed. The blood THg concentration $\times$ study interaction was non-significant $\left(F_{2,89.1}=0.08, p=0.92\right)$, indicating that the relationship between THg concentrations in eggs and blood had a similar slope among the three mallard studies and we removed this interaction from the model. THg concentrations in eggs were positively correlated with THg concentrations in the mother's blood in mallards $\left(F_{1,45.8}=330.64\right.$, $p<0.0001)$, but there was an influence of study $\left(F_{2,80.4}=6.07, p=0.004\right.$; final model: $n=255$, $R_{m}^{2}=0.87$; Figure 1c). The data derived from dosed captive mallards (Eqs. 8 and 9) resulted in greater predicted egg THg concentrations than wild mallards (Eq. 10) at any given female blood $\mathrm{THg}$ concentration. Also noteworthy was that there were no significant differences in the modeled relationships between the two captive mallard datasets, ${ }^{4}$ despite the difference in the timing of female blood sampling between studies where females were bled either on the day of egg laying or after she had laid an additional 16-27 eggs (which was approximately 16-27 days after the initial egg was laid that was assessed for THg concentrations). The study-specific equations to predict $\mathrm{THg}$ concentrations in eggs from maternal blood were (Table S2):

(8) $\ln \left(\right.$ Egg THg $\left.\frac{\mu g}{g} f w w\right)=0.8837 \times \ln \left(\right.$ Female Mallard Blood THg $\left.g_{\frac{\mu g}{g} w w}\right)+0.0878$ (captive females bled on day of egg laying, ${ }^{4} n=15$ eggs, $R_{m}^{2}=0.89$ )

(9) $\ln \left(\right.$ Egg THg $\left.\frac{\mu g}{g} f w w\right)=0.8140 \times \ln \left(\right.$ Female Mallard Blood THg $\left.\frac{\mu g}{g} w w\right)+0.1075$ (captive females bled after laying an additional $16-27$ eggs, ${ }^{4} n=15$ eggs, $R_{m}^{2}=0.83$ )

(10) $\ln \left(E g g T H g_{\frac{\mu g}{g} f w w}\right)=0.8690 \times \ln \left(\right.$ Female Mallard Blood THg $\left.g_{\frac{\mu g}{g} w w}\right)-0.2574$ (Evers, wild females bled prior to and during incubation, $n=225$ eggs, $R_{m}^{2}=0.83$ ) 


\section{Carolina wren}

We had two unique datasets for Carolina wrens. Due to the limited sample size for Carolina wrens ( $n=6$ nests), we could not include the blood THg concentration $\times$ study interaction. THg concentrations in eggs were positively correlated with THg concentrations in the mother's blood in Carolina wrens $\left(F_{1,3.2}=66.36, p=0.003\right)$ and there was no influence of study ( $F_{1,1.7}=0.02, p=0.91$; final model: $n=9, R_{m}^{2}=0.88$; Figure $\left.1 \mathrm{e}\right)$. The study-specific equations between THg concentrations in eggs and maternal blood were (Table S2):

(11) $\ln \left(\right.$ Egg THg $\left.{ }_{\frac{\mu g}{g}} f w w\right)=0.7736 \times \ln \left(\right.$ Female Carolina Wren Blood THg $\left.\frac{\mu g}{g} w w\right)-1.9399$ (Cristol, South River, Shenandoah Valley, Virginia, $n=4$ eggs, $R_{m}^{2}=0.99$ )

(12) $\ln \left(\right.$ Egg THg $\left.\operatorname{sig}_{g} f w w\right)=0.4801 \times \ln \left(\right.$ Female Carolina Wren Blood THg $\left.{ }_{\frac{\mu g}{g} w w}\right)-1.9153$ (Evers, North Fork Holston River, Virginia, $n=5$ eggs, $R_{m}^{2}=0.54$ )

\section{Black-legged kittiwake}

We had two unique datasets for black-legged kittiwakes where the geographic location differed between studies. After removing the non-significant blood $\mathrm{THg}$ concentration $\times$ study interaction term $\left(F_{1,11}=2.38, p=0.15\right)$, THg concentrations in eggs were positively correlated with THg concentrations in the mother's blood in black-legged kittiwakes $\left(F_{1,12}=27.40, p=0.0002\right)$, but there was an influence of study $\left(F_{1,12}=12.53, p=0.004\right.$; final model: $n=15, R_{m}^{2}=0.81$; Figure 1d). In particular, the larger dataset for black-legged kittiwakes from Corossol Island, Quebec (Eq. 14) had much higher explanatory power than the smaller dataset from Prince Leopold Island, Nunavut (Eq. 13). The study-specific equations to predict THg concentrations in eggs from maternal blood were (Table S2):

(13) $\ln \left(\right.$ Egg THg $\left.\frac{\mu g}{g} f w w\right)=0.2751 \times \ln \left(\right.$ Female Black - legged Kittiwake Blood THg $\left.\frac{\mu g}{g} w w\right)-1.9807$ (Mallory, Provencher, Braune; Prince Leopold Island, Nunavut, $n=5$ eggs, $R_{m}^{2}=0.27$ )

(14) $\ln \left(\right.$ Egg THg $\left.g_{\frac{\mu g}{g} f w w}\right)=1.2601 \times \ln \left(\right.$ Female Black - legged Kittiwake Blood THg $\left.\frac{\mu g}{g} w w\right)+0.1930$ (Lavoie, Corossol Island, Quebec, $n=10$ eggs, $R_{m}^{2}=0.73$ ) 


\section{Egg to maternal blood relationship: by species}

Second, we examined whether the relationship between THg concentrations in eggs and maternal blood differed among species. Because some species differed in their relationships within taxonomic orders, we conducted separate models for each species to estimate the specific equations to predict THg concentrations in eggs from THg concentrations in maternal blood (Figure 3c).

(15) $\ln \left(\right.$ Egg THg $\left.g_{\frac{\mu g}{g} f w w}\right)=0.9295 \times \ln \left(\right.$ Female American Avocet Blood THg $\left.\frac{\mu \underline{g}}{g} w w_{w}\right)-0.8965(n=97$ eggs, $\left.R_{m}^{2}=0.73\right)$

(16) $\ln \left(\right.$ Egg THg $\left.g_{\frac{\mu g}{g} f w w}\right)=0.8602 \times \ln \left(\right.$ Female Belted Kingfisher Blood THg $\left.\frac{\underline{\mu} g}{g w w}\right)-2.4879(n=24$ eggs, $\left.R_{m}^{2}=0.87\right)$

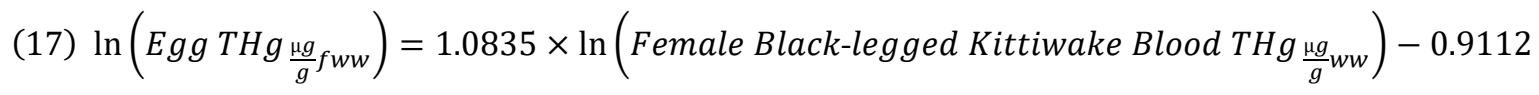
( $n=15$ eggs, $\left.R_{m}^{2}=0.49\right)$

(18) $\ln \left(\right.$ Egg THg $\left.g_{\frac{\mu g}{g} f w w}\right)=0.7225 \times \ln \left(\right.$ Female Black-necked Stilt Blood THg $\left.\frac{\mu g}{g}_{w w}\right)-0.7075(n=105$ eggs, $R_{m}^{2}=0.69$ )

(19) $\ln \left(\right.$ Egg THg $\left.\frac{\mu g}{g} f w w\right)=0.7090 \times \ln \left(\right.$ Female Carolina Wren Blood THg $\left.g_{\frac{\mu g}{g} w w}\right)-1.9443(n=9$ eggs, $\left.R_{m}^{2}=0.90\right)$

(20) $\ln \left(\right.$ Egg THg $\left._{\frac{\mu g}{g} f w w}\right)=0.8531 \times \ln \left(\right.$ Female Common Eider Blood THg $\left.g_{\frac{\mu g}{g} w w}\right)+0.2416(n=18$ eggs, $\left.R_{m}^{2}=0.64\right)$

(21) $\ln \left(\right.$ Egg THg $\left.\frac{\underline{\underline{g}}}{g} f w w\right)=0.2827 \times \ln \left(\right.$ Female Common Goldeneye Blood THg $\left.\frac{\underline{\mu}}{g} w w\right)-1.0441(n=11$ eggs, $\left.R_{m}^{2}=0.01\right)$

(22) $\ln \left(\right.$ Egg THg $\left.\frac{\underline{\mu} g}{g} f w w\right)=0.7218 \times \ln \left(\right.$ Female Common Loon Blood THg $\left.\frac{\underline{\mu} g}{g} w w\right)-0.6271(n=119$ eggs, $\left.R_{m}^{2}=0.47\right)$

(23) $\ln \left(\right.$ Egg THg $\left.\frac{\mu g}{g} f w w\right)=0.5618 \times \ln \left(\right.$ Female Common Merganser Blood THg $\left.g_{\frac{\mu g}{g} w w}\right)-0.4308(n=5$ eggs, $\left.R_{m}^{2}=0.29\right)$

(24) $\ln \left(\right.$ Egg THg $\left._{\frac{\underline{\mu}}{g} f w w}\right)=1.9391 \times \ln \left(\right.$ Female Eastern Bluebird Blood THg $\left.\frac{\underline{\underline{\mu g}}}{g w w}\right)-1.2038(n=5$ eggs, $\left.R_{m}^{2}=0.71\right)$ 
(25) $\ln \left(\right.$ Egg THg $\left.\frac{\mu g}{g} f w w\right)=0.7785 \times \ln \left(\right.$ Female Forster's Tern Blood THg $\left.\frac{\mu g}{g}_{w w}\right)-0.5465(n=49$ eggs, $\left.R_{m}^{2}=0.26\right)$

(26) $\ln \left(\right.$ Egg THg $\left.\frac{\mu g}{g} f w w\right)=1.3734 \times \ln \left(\right.$ Female Herring Gull Blood THg $\left.{ }_{\frac{\mu g}{g} w w}\right)+0.6525(n=12$ eggs, $\left.R_{m}^{2}=0.67\right)$

(27) $\ln \left(E g g T H g_{\frac{\mu g}{g} f w w}\right)=0.3425 \times \ln \left(\right.$ Female Hooded Merganser Blood THg $\left.g_{\frac{\mu g}{g} w w}\right)-0.4600(n=34$ eggs, $R_{m}^{2}=0.22$ )

(28) $\ln \left(\right.$ Egg THg $\left.g_{\frac{\mu g}{g} f w w}\right)=1.0444 \times \ln \left(\right.$ Female House Wren Blood THg $\left.\frac{\mu g}{g} w w\right)-1.6690(n=306$ eggs, $R_{m}^{2}=0.82$ )

(29) $\ln \left(\right.$ Egg THg $\left.\frac{\mu \underline{g}}{g} f w w\right)=0.8263 \times \ln \left(\right.$ Female Indigo Bunting Blood THg $\left.\frac{\mu g}{g} w w\right)-2.3433(n=23$ eggs, $\left.R_{m}^{2}=0.78\right)$

(30) $\ln \left(\right.$ Egg THg $\left.\frac{\mu g}{g} f w w\right)=0.8802 \times \ln \left(\right.$ Female Mallard Blood THg $\left.\frac{\mu g}{g} w w\right)-0.0892(n=240$ eggs, $\left.R_{m}^{2}=0.81\right)$

(31) $\ln \left(\right.$ Egg THg $\left.\frac{\mu g}{g} f w w\right)=0.3227 \times \ln \left(\right.$ Female Northern Fulmar Blood THg $\left.\frac{\mu g}{g} w w\right)-1.3870(n=3$ eggs, $\left.R_{m}^{2}=0.17\right)$

(32) $\ln \left(\right.$ Egg THg $\left.g_{\frac{\mu g}{g} f w w}\right)=1.0289 \times \ln \left(\right.$ Female Song Sparrow Blood THg $\left.g_{\frac{\mu g}{g} w w}\right)-1.2475(n=25$ eggs, $\left.R_{m}^{2}=0.87\right)$

(33) $\ln \left(\right.$ Egg THg $\left.\frac{\mu g}{g} f w w\right)=0.8327 \times \ln \left(\right.$ Female Tree Swallow Blood THg $\left.\frac{\mu g}{g} w w\right)-1.2819(n=568$ eggs, $\left.R_{m}^{2}=0.87\right)$

(34) $\ln \left(\right.$ Egg THg $\left.\operatorname{sg}_{g} f w w\right)=0.6007 \times \ln \left(\right.$ Female Wood Duck Blood THg $\left.\frac{\mu g}{g} w w\right)-1.3460(n=9$ eggs, $\left.R_{m}^{2}=0.11\right)$

(35) $\ln \left(\right.$ Egg THg $\left.\frac{\mu g}{g} f w w\right)=0.6472 \times \ln \left(\right.$ Female Yellow - billed Loon Blood THg $\left.g_{\frac{\mu g}{g} w w}\right)-0.7751(n=6$ eggs, $\left.R_{m}^{2}=0.50\right)$

(36) $\ln \left(\right.$ Egg THg $\left.g_{\frac{\mu g}{g} f w w}\right)=0.7133 \times \ln \left(\right.$ Female Zebra Finch Blood THg $\left.\frac{\mu g}{g} w w_{w}\right)-0.2478(n=104$ eggs, $\left.R_{m}^{2}=0.64\right)$ 


\section{REFERENCES}

(1) Ackerman, J. T.; Eagles-Smith, C. A.; Herzog, M. P.; Hartman, C. A. Maternal Transfer of Contaminants in Birds: Mercury and Selenium Concentrations in Parents and Their Eggs. Environ. Pollut. 2016, 210, 145-154.

(2) Brasso, R. L.; Abdel Latif, M. K.; Cristol, D. A. Relationship between Laying Sequence and Mercury Concentration in Tree Swallow Eggs. Environ. Toxicol. Chem. 2010, 29 (5), 1155-1159. https://doi.org/10.1002/etc.144.

(3) Ackerman, J. T.; Hartman, C. A.; Herzog, M. P. Maternal Transfer of Mercury to Songbird Eggs. Environ. Pollut. 2017, 230, 463-468.

https://doi.org/10.1016/j.envpol.2017.06.099.

(4) Heinz, G. H.; Hoffman, D. J.; Klimstra, J. D.; Stebbins, K. R. Predicting Mercury Concentrations in Mallard Eggs from Mercury in the Diet or Blood of Adult Females and from Duckling down Feathers. Environ. Toxicol. Chem. 2010, 29 (2), 389-392. https://doi.org/10.1002/etc.50.

(5) Evers, D. C.; Taylor, K. M.; Major, A.; Taylor, R. J.; Poppenga, R. H.; Scheuhammer, A. M. Common Loon Eggs as Indicators of Methylmercury Availability in North America. Ecotoxicology 2003, 12 (1-4), 69-81.

(6) Lavoie, R. A.; Hebert, C. E.; Rail, J. F.; Braune, B. M.; Yumvihoze, E.; Hill, L. G.; Lean, D. R. S. Trophic Structure and Mercury Distribution in a Gulf of St. Lawrence (Canada) Food Web Using Stable Isotope Analysis. Sci. Total Environ. 2010, 408 (22), 5529-5539. https://doi.org/10.1016/j.scitotenv.2010.07.053.

(7) Kenow, K. P.; Meyer, M. W.; Rossmann, R.; Gray, B. R.; Arts, M. T. Influence of in Ovo Mercury Exposure, Lake Acidity, and Other Factors on Common Loon Egg and Chick Quality in Wisconsin. Environ. Toxicol. Chem. 2015, 34 (8), 1870-1880. https://doi.org/10.1002/etc.3001.

(8) Mallory, M. L.; Provencher, J. F.; Robertson, G. J.; Braune, B. M.; Holland, E. R.; Klapstein, S.; Stevens, K.; Driscoll, N. J. O. Mercury Concentrations in Blood, Brain and Muscle Tissues of Coastal and Pelagic Birds from Northeastern Canada. Ecotoxicol. Environ. Saf. 2018, 157, 424-430. https://doi.org/10.1016/j.ecoenv.2018.04.004.

(9) Nakagawa, S.; Schielzeth, H. A General and Simple Method for Obtaining R2 from Generalized Linear Mixed-Effects Models. Methods Ecol. Evol. 2013, 4 (2), 133-142. 
https://doi.org/10.1111/j.2041-210x.2012.00261.x.

(10) Nakagawa, S.; Johnson, P. C. D.; Schielzeth, H. The Coefficient of Determination R2 and Intra-Class Correlation Coefficient from Generalized Linear Mixed-Effects Models Revisited and Expanded. Interface 2017, 14, 20170213.

(11) Johnston, T. A.; Bodaly, R. A.; Latif, M. A.; Fudge, R. J. P.; Strange, N. E. Intra- and Interpopulation Variability in Maternal Transfer of Mercury to Eggs of Walleye (Stizostedion Vitreum). Aquat. Toxicol. 2001, 52 (1), 73-85. https://doi.org/10.1016/S0166-445X(00)00129-6.

(12) Kenow, K. P.; Meyer, M. W.; Rossmann, R.; Gray, B. R.; Arts, M. T. Influence of in Ovo Mercury Exposure, Lake Acidity, and Other Factors on Common Loon Egg and Chick Quality in Wisconsin. Environ. Toxicol. Chem. 2015, 34 (8), 1870-1880. https://doi.org/10.1002/etc.3001. 


\section{TABLES}

Table S1. Summary of available datasets on the maternal transfer of mercury from females to her eggs in 26 species and 6 taxonomic orders of birds in North America. Table S1 is an extension of Table 1 in the main manuscript and includes additional details about the methods used to estimate blood and egg total mercury concentrations (THg) and the timing of egg and female blood sampling for each dataset (denoted by the citation and species). 


\begin{tabular}{|c|c|c|}
\hline Citation & Common Name & $\begin{array}{l}\text { Female Blood THg Estimation } \\
\text { Method }\end{array}$ \\
\hline $\begin{array}{l}\text { Ackerman et al. } \\
2016 \mathrm{a}\end{array}$ & American avocet & whole blood in wet weight \\
\hline $\begin{array}{l}\text { Ackerman et al. } \\
2016 \mathrm{a}\end{array}$ & Black-necked stilt & whole blood in wet weight \\
\hline $\begin{array}{l}\text { Ackerman et al. } \\
\text { 2016a }\end{array}$ & Forster's tern & whole blood in wet weight \\
\hline $\begin{array}{l}\text { Ackerman et al. } \\
2017\end{array}$ & Tree swallow & $\begin{array}{l}\text { whole blood in wet weight: } \\
\text { estimated using whole blood in dry } \\
\text { weight with specific percent } \\
\text { mositure to calculate wet weight }\end{array}$ \\
\hline $\begin{array}{l}\text { Ackerman et al. } \\
2017\end{array}$ & House wren & $\begin{array}{l}\text { whole blood in wet weight: } \\
\text { estimated using whole blood in dry } \\
\text { weight with specific percent } \\
\text { mositure to calculate wet weight }\end{array}$ \\
\hline $\begin{array}{l}\text { Ackerman et al. } \\
2017\end{array}$ & Tree swallow & $\begin{array}{l}\text { whole blood in wet weight: } \\
\text { estimated using whole blood in dry } \\
\text { weight with specific percent } \\
\text { mositure to calculate wet weight }\end{array}$ \\
\hline Kenow et al. 2015 & Common loon & whole blood in wet weight \\
\hline Heinz et al. 2010b & Mallard & whole blood in wet weight \\
\hline Heinz et al. 2010b & Mallard & whole blood in wet weight \\
\hline $\begin{array}{l}\text { Mallory, } \\
\text { Provencher, } \\
\text { Braune; this paper }\end{array}$ & Northern fulmar & $\begin{array}{l}\text { whole blood in wet weight: } \\
\text { estimated using whole blood in dry } \\
\text { weight; used } 79.1 \% \text { (Eagles-Smith } \\
\text { et al. 2008) average percent } \\
\text { mositure to calculate THg in wet } \\
\text { weight }\end{array}$ \\
\hline
\end{tabular}

Egg THg Estimation Method

resh wet weight: estimated from dry weight using individual egg's percent moisture and egg morphometrics

fresh wet weight: estimated from dry weight using individual egg's percent moisture and egg morphometrics

fresh wet weight: estimated from dry weight using individual egg's percent moisture and egg morphometrics

fresh wet weight: estimated from dry weight using individual egg's percent moisture and egg morphometrics

fresh wet weight: estimated from dry weight using individual egg's percent moisture and egg

morphometrics

fresh wet weight: estimated from dry weight using individual egg's percent moisture and egg morphometrics

fresh wet weight: estimated from dry weight using individual egg's percent moisture and egg morphometrics

wet weight; no egg morphometrics; assumed $\mathrm{THg}$ fww $=\mathrm{THg}$ ww because eggs were collected fresh on day they were laid

wet weight; no egg morphometrics; assumed $\mathrm{THg}$ fww $=\mathrm{THg}$ ww because eggs were collected fresh on day they were laid

fresh wet weight: estimated from dry weight using individual egg's

percent moisture and egg morphometrics
Timing of egg sampling eggs were collected during early incubation, on same day when female blood was collected

eggs were collected during early incubation, on same day when female blood was collected

eggs were collected during early incubation, on same day when female blood was collected

eggs were collected immediately after clutch completion, on same day when female blood was collected

eggs were collected immediately after clutch completion, on same day when female blood was collected

egg was collected during late incubation, on same day when female blood was collected

eggs were collected during early incubation, on same day when female blood was collected

egg was collected on day it was laid, on same day when female blood was collected

egg was collected on day it was laid, which was 16-27 days before female blood was collected

eggs were collected during incubation, on same day when female was collected
Timing of female blood sampling female captured on nest and bled during early incubation, $\leq 12$ days after clutch completion

female captured on nest and bled during early incubation, $\leq 12$ days after clutch completion

female captured on nest and bled during early incubation, $\leq 12$ days after clutch completion

female captured on nest and bled $\leq 3$ days after clutch completion

female captured on nest and bled $\leq 3$ days after clutch completion

female captured on nest and bled during late incubation, 6-10 days after clutch completion

female captured on nest and bled $\leq 8$ days after clutch completion

female captured on nest and bled the day the single egg was collected, egg was one of 33 to 44 eggs laid in a clutch

female captured on nest and bled 16 to 27 days after egg was laid

female was collected $\leq 28$ days after clutch completion, frozen, and then blood was sampled from heart during necropsy 


\begin{tabular}{|c|c|c|}
\hline Mallory, & Black-legged & whole blood in wet weight: \\
\hline $\begin{array}{l}\text { Provencher, } \\
\text { Braune; this paper }\end{array}$ & kittiwake & $\begin{array}{l}\text { estimated using whole blood in dry } \\
\text { weight; used } 79.1 \% \text { (Eagles-Smith }\end{array}$ \\
\hline & & $\begin{array}{l}\text { et al. } 2008 \text { ) average percent } \\
\text { mositure to calculate THg in wet } \\
\text { weight }\end{array}$ \\
\hline Lavoie; this paper & Herring gull & $\begin{array}{l}\text { whole blood in wet weight: } \\
\text { estimated using red blood cells in } \\
\text { dry weight, converting to wet } \\
\text { weight, then estimating whole } \\
\text { blood wet weight based on ratio of } \\
\text { red blood cells to plasma }\end{array}$ \\
\hline Lavoie; this paper & $\begin{array}{l}\text { Black-legged } \\
\text { kittiwake }\end{array}$ & $\begin{array}{l}\text { whole blood in wet weight: } \\
\text { estimated using red blood cells in } \\
\text { dry weight, converting to wet } \\
\text { weight, then estimating whole } \\
\text { blood wet weight based on ratio of } \\
\text { red blood cells to plasma }\end{array}$ \\
\hline Lavoie; this paper & Common eider & $\begin{array}{l}\text { whole blood in wet weight: } \\
\text { estimated using red blood cells in } \\
\text { dry weight, converting to wet } \\
\text { weight, then estimating whole } \\
\text { blood wet weight based on ratio of } \\
\text { red blood cells to plasma }\end{array}$ \\
\hline Lavoie; this paper & $\begin{array}{l}\text { Great black-backed } \\
\text { gull }\end{array}$ & $\begin{array}{l}\text { whole blood in wet weight: } \\
\text { estimated using red blood cells in } \\
\text { dry weight, converting to wet } \\
\text { weight, then estimating whole } \\
\text { blood wet weight based on ratio of } \\
\text { red blood cells to plasma }\end{array}$ \\
\hline
\end{tabular}

Brasso et al. $2010 \quad$ Tree swallow whole blood in wet weight

Cristol; this paper Zebra finch

whole blood in wet weight

Cristol; this paper Tree swallow fresh wet weight: estimated from dry weight using individual egg's percent moisture and egg morphometrics

fresh wet weight: estimated from dry weight using individual egg's percent moisture and egg morphometrics

fresh wet weight: estimated from dry weight using individual egg's percent moisture and egg morphometrics

fresh wet weight: estimated from dry weight using individual egg's percent moisture and egg morphometrics

fresh wet weight: estimated from dry weight using individual egg's percent moisture and egg morphometrics

wet weight: estimated from dry weight using individual egg's percent moisture; no egg morphometrics; assumed THg fww $=\mathrm{THg} \mathrm{ww}$

wet weight: estimated from dry weight assuming an average percent moisture of $75.4 \%$ in eggs; no egg morphometrics; assumed $\mathrm{THg}$ fww $=\mathrm{THg} \mathrm{ww}$

wet weight: estimated from dry weight using individual egg's percent moisture; no egg morphometrics; assumed THg fww $=\mathrm{THg} \mathrm{ww}$ egg was collected during incubation, on same day when female was collected

egg was collected during incubation, on same day when female blood was collected

egg was collected during incubation, on same day when female blood was collected

egg was collected during incubation, on same day when female blood was collected

egg was collected during incubation, on same day when female blood was collected

eggs were collected immediately after clutch completion, on same day when female blood was day when
collected

eggs were collected during incubation, on same day when female blood was collected

eggs were collected during incubation, on same day when female blood was collected female was collected $\leq 10$ days after clutch completion, frozen, and then blood was sampled from heart during necropsy

female captured on nest and bled $\leq 28$ days after clutch completion

female captured on nest and bled $\leq 27$ days after clutch completion

female captured on nest and bled $\leq 26$ days after clutch completion

female captured on nest and bled $\leq 28$ days after clutch completion

female captured on nest and bled $\leq 3$ days after clutch completion

female captured on nest and bled $\leq 7$ days after clutch completion

female captured on nest and bled $\leq 7$ days after clutch completion 
whole blood in wet weight

Cristol; this paper

Belted kingfisher

whole blood in wet weight

Evers; this pape

Carolina wren

whole blood in wet weight

Evers et al. 2003, and additiona

unpublished data

for this paper

Evers; this paper

Indigo bunting

whole blood in wet weight

Evers; this paper

Northern cardinal

whole blood in wet weight

Evers; this paper
Red-winged

blackbird wet weight: estimated from dry weight using individual egg's percent moisture; no eg

morphometrics; assumed THg fww $=\mathrm{THg} \mathrm{ww}$

wet weight: estimated from dry weight using individual egg's

percent moisture; no egg

morphometrics; assumed THg fww $=\mathrm{THg} w \mathrm{w}$

wet weight: estimated from dry weight using individual egg's percent moisture; no egg morphometrics; assumed THg fww = THg ww

wet weight: estimated from dry weight using individual egg's percent moisture; no eg morphometrics; assumed THg fww $=\mathrm{THg} \mathrm{ww}$

wet weight: estimated from dry weight using individual egg's percent moisture; no egg morphometrics; assumed THg fww $=\mathrm{THg} \mathrm{ww}$

wet weight; no egg morphometrics; assumed THg fww $=\mathrm{THg}$ ww

fresh wet weight: estimated from either 1) dry weight using individual egg's percent moisture and egg morphometrics or 2) wet weight and individual egg morphometrics

wet weight: estimated from dry weight using individual egg's percent moisture; no eg. morphometrics; assumed THg fww = THg ww

wet weight: estimated from dry weight using individual egg's percent moisture; no egg morphometrics; assumed THg fww $=\mathrm{THg}$ ww

wet weight: estimated from dry weight using individual egg's

percent moisture; no egg

morphometrics; assumed THg fww $=\mathrm{THg} w \mathrm{w}$ egg was collected during ncubation, on same day when female blood was collected

eggs were collected during incubation, on same day when female blood was collected

eggs were collected during incubation, on same day when female blood was collected

egg was collected during incubation, on same day when female blood was collected

eggs were collected during incubation, on same day when female blood was collected

egg was collected during incubation, $\leq 6$ days after when female blood was collected

eggs were collected from

abandoned nests, $\leq 69$ days after egg laying

eggs were collected during

incubation, $\leq 3$ days of when female blood was collected

eggs were collected during incubation, on same day whe female blood was collected

eggs were collected during incubation, on same day when female blood was collected female captured on nest and bled $\leq 7$ days after clutch completion

female captured on nest and bled $\leq 7$ days after clutch completion

female captured on nest and bled $\leq 7$ days after clutch completion

female captured on nest and bled $\leq 7$ days after clutch completion

female captured on nest and bled $\leq 7$ days after clutch completion

female captured on nest and bled $\leq 6$ days before egg collection

female captured in territory and bled $\leq 69$ days before or after clutch completion

female captured on nest and bled $\leq 16$ days after clutch completion

female captured on nest and bled $\leq 13$ days after clutch completion

female captured on nest and bled $\leq 12$ days after clutch completion 
whole blood in wet weight

Evers, Savoy; this paper

Evers, Savoy; this paper

Evers, Savoy; this paper

Evers, Savoy; this paper

Evers, Savoy; this paper

Matz, Schmutz; this paper

\section{Common} goldeneye

Common merganser

\section{Hooded merganser}

Mallard

whole blood in wet weight

\section{Wood duck}

whole blood in wet weight

whole blood in wet weight: estimated using whole blood in dry weight; used $75.9 \%$ (Ackerman et al. 2015) average percent mositure to calculate $\mathrm{THg}$ in wet weight

wet weight: estimated from dry weight using individual egg's percent moisture; no egg morphometrics; assumed THg fww $=\mathrm{THg} \mathrm{ww}$

wet weight: estimated from dry weight using individual egg's percent moisture; no egg morphometrics; assumed THg fww $=\mathrm{THg} \mathrm{ww}$

fresh wet weight: estimated from dry weight using individual egg's percent moisture and egg morphometrics

fresh wet weight: estimated from dry weight using individual egg's percent moisture and egg morphometrics

fresh wet weight or wet weight: estimated from either 1) dry weig using individual egg's percent moisture and egg morphometrics or 2 ) wet weight and assumed $\mathrm{TH}$ $\mathrm{fww}=\mathrm{THg} \mathrm{ww}$

fresh wet weight: estimated from either 1) dry weight using individual egg's percent moisture and egg morphometrics or 2) wet weight and individual egg morphometrics fresh wet weight or wet weight: estimated from either 1) wet weight and individual egg morphometrics or 2) wet weigh and assumed $\mathrm{THg}$ fww $=\mathrm{THg}$ ww

wet weight: estimated from dry weight assuming an average percent moisture of $75.5 \%$

(Ackerman et al. 2015) in eggs; no egg morphometrics; assumed $\mathrm{TH}$ eggs were collected during fame day when female blood was collected

egg was collected during incubation, $\leq 16$ days before when female blood was collected

eggs were collected from

abandoned nests, $\leq 12$ days after when female blood was collected

eggs were collected from abandoned nests, $\leq 28$ days before or after after when female blood was collected

eggs were collected from

abandoned nests, $\leq 28$ days before or after after when female blood was collected

eggs were collected during incubation, $\leq 20$ days of when female blood was collected

egg was collected during incubation, on same day when female blood was collected

egg was collected during incubation, on same day when female blood was collected female captured on nest and bled $\leq 14$ days after clutch completion

female captured on nest and bled $\leq 28$ days after clutch completion

female captured on nest and bled $\leq 12$ days before egg collection

female captured in territory and bled $\leq 28$ days before or after clutch completion

female captured in territory and bled $\leq 28$ days before or after clutch completion

female captured, bled,

transmittered, and than followed to locate nest; female bled $\leq 20$ days before clutch completion

female captured on nest and bled $\leq 28$ days after clutch completion

female captured on nest and bled $\leq 28$ days after clutch completion 
Table S2. Equations (both on the natural log scale and back-transformed), model fit, and variance for predicting egg total mercury concentrations ( $\mathrm{THg} \mu \mathrm{g} / \mathrm{g}$ fww) based on female blood $\mathrm{THg}$ concentrations $(\mu \mathrm{g} / \mathrm{g} \mathrm{ww})$ for all taxa, 6 taxonomic orders, 22 species of birds, and by study. The equation number from the text is provided for reference. Because there were no other fixed-effects in the models, marginal $R_{m}^{2}$ values indicated the explanatory power of $\mathrm{THg}$ concentrations in female bird blood for predicting THg concentrations in eggs. Conditional $R_{c}^{2}$ values described the proportion of variance explained by both the fixed- and random-effects in the model. 


\begin{tabular}{|c|c|c|c|c|c|c|c|c|}
\hline Taxa & $\begin{array}{r}\text { Sample } \\
\text { Size of } \\
\text { Eggs } \\
\end{array}$ & $R_{c}^{2}$ & $R_{m}^{2}$ & $\begin{array}{r}\text { Slope } \pm \text { SE } \\
\text { (log scale) }\end{array}$ & $\begin{array}{r}\text { Intercept } \pm \text { SE } \\
(\log \text { scale) }\end{array}$ & $\begin{array}{r}\text { Equation } \\
\text { Reference } \\
\text { Number } \\
\end{array}$ & Equation (log scale) & Equation (back-transformed) \\
\hline All taxa & 1799 & 0.97 & 0.58 & $0.8220 \pm 0.0190$ & $-0.9947 \pm 0.1441$ & 42 & $\ln (\mathrm{Egg} \mathrm{THg})=0.8220 \times \ln ($ Female Blood THg $)-0.9947$ & Egg THg $=0.3698 \times$ Female Blood $\mathrm{THg}^{0.8220}$ \\
\hline Anseriformes & 317 & 0.91 & 0.72 & $0.7661 \pm 0.0564$ & $-0.2470 \pm 0.1994$ & 37 & $\ln (\mathrm{Egg} \mathrm{THg})=0.7661 \times \ln ($ Female Blood THg $)-0.2470$ & Egg THg $=0.7811 \times$ Female Blood $\mathrm{THg}^{0.7661}$ \\
\hline Common eider & 18 & 0.64 & 0.64 & $0.8531 \pm 0.1548$ & $0.2416 \pm 0.3812$ & 20 & $\ln ($ Egg THg $)=0.8531 \times \ln ($ Female Blood THg $)+0.2416$ & Egg THg $=1.2733 \times$ Female Blood $\mathrm{THg}^{0.8331}$ \\
\hline Common goldeneye & 11 & 0.61 & 0.01 & $0.2827 \pm 1.8426$ & $-1.0441 \pm 2.7439$ & 21 & $\ln ($ Egg THg $)=0.2827 \times \ln ($ Female Blood THg $)-1.0441$ & Egg THg $=0.3520 \times$ Female Blood $\mathrm{THg}^{0.2227}$ \\
\hline Common merganser & 5 & 0.98 & 0.29 & $0.5618 \pm 0.5306$ & $-0.4308 \pm 0.1859$ & 23 & $\ln ($ Egg THg $)=0.5618 \times \ln ($ Female Blood THg $)-0.4308$ & Egg THg $=0.6500 \times$ Female Blood THg ${ }^{0.5618}$ \\
\hline Hooded merganser & 34 & 0.22 & 0.22 & $0.3425 \pm 0.1125$ & $-0.4600 \pm 0.1088$ & 27 & $\ln ($ Egg THg $)=0.3425 \times \ln ($ Female Blood THg $)-0.4600$ & Egg THg $=0.6313 \times$ Female Blood THg ${ }^{0.3425}$ \\
\hline Heinz et al. 2010; captive females bled on day of egg laying & 15 & 0.89 & 0.89 & $0.8837 \pm 0.0840$ & $0.0878 \pm 0.0696$ & 8 & $\ln ($ Egg THg $)=0.8837 \times \ln ($ Female Blood THg $)+0.0878$ & Egg THg $=1.0918 \times$ Female Blood THg ${ }^{0.837}$ \\
\hline Heinz et al. 2010 ; captive females bled 16-27 days after egg laying & 15 & 0.83 & 0.83 & $0.8140 \pm 0.0996$ & $0.1075 \pm 0.0825$ & 9 & $\ln ($ Egg THg $)=0.8140 \times \ln ($ Female Blood THg $)+0.1075$ & Egg THg $=1.1135 \times$ Female Blood $\mathrm{THg}^{0.8140}$ \\
\hline Evers; wild females bled prior to and during incubation & 225 & 0.92 & 0.83 & $0.8690 \pm 0.0651$ & $-0.2574 \pm 0.0788$ & 10 & $\ln ($ Egg THg $)=0.8690 \times \ln ($ Female Blood THg $)-0.2574$ & Egg THg $=0.7731 \times$ Female Blood THg ${ }^{0.8690}$ \\
\hline Wood duck & 9 & 0.99 & 0.11 & $0.6007 \pm 0.5956$ & $-1.3460 \pm 1.9506$ & 34 & $\ln ($ Egg THg $)=0.6007 \times \ln ($ Female Blood THg $)-1.3460$ & Egg THg $=0.2603 \times$ Female Blood THg ${ }^{0.6007}$ \\
\hline Charadriiformes & 280 & 0.95 & 0.71 & $0.8761 \pm 0.0569$ & $-0.7961 \pm 0.1742$ & 38 & $\ln (\mathrm{Egg} \mathrm{THg})=0.8761 \times \ln ($ Female Blood THg $)-0.7961$ & Egg THg $=0.4511 \times$ Female Blood $\mathrm{THg}^{0.8761}$ \\
\hline American avocet & 97 & 0.94 & 0.73 & $0.9295 \pm 0.0995$ & $-0.8965 \pm 0.1003$ & 15 & $\ln ($ Egg THg $)=0.9295 \times \ln ($ Female Blood THg $)-0.8965$ & Egg THg $=0.4080 \times$ Female Blood THg ${ }^{0.9295}$ \\
\hline Black-legged kittiwake & 15 & 0.98 & 0.49 & $1.0835 \pm 0.2438$ & $-0.9112 \pm 0.7970$ & 17 & $\ln (\mathrm{Egg} \mathrm{THg})=1.0835 \times \ln ($ Female Blood THg $)-0.9112$ & Egg THg $=0.4020 \times$ Female Blood $\mathrm{THg}^{1.0835}$ \\
\hline Mallory, Provencher, Braune; Prince Leopold Island, Nunavut & 5 & 0.27 & 0.27 & $0.2751 \pm 0.2277$ & $-1.9807 \pm 0.1106$ & 13 & $\ln ($ Egg THg $)=0.2751 \times \ln ($ Female Blood THg $)-1.9807$ & Egg THg $=0.1380 \times$ Female Blood $\mathrm{THg}^{0.2751}$ \\
\hline Black-necked stilt & 105 & 0.85 & 0.69 & $0.7225 \pm 0.0720$ & $-0.7075 \pm 0.0475$ & 18 & $\ln ($ Egg THg $)=0.7225 \times \ln ($ Female Blood THg $)-0.7075$ & Egg THg $=0.4929 \times$ Female Blood THg ${ }^{0.7225}$ \\
\hline Forster's tern & 49 & 0.78 & 0.26 & $0.7785 \pm 0.2788$ & $-0.5465 \pm 0.2216$ & 25 & $\ln ($ Egg THg $)=0.7785 \times \ln ($ Female Blood THg $)-0.5465$ & Egg THg $=0.5790 \times$ Female Blood THg ${ }^{0.7785}$ \\
\hline Herring gull & 12 & 0.67 & 0.67 & $1.3734 \pm 0.2916$ & $0.6525 \pm 0.6508$ & 26 & $\ln ($ Egg THg $)=1.3734 \times \ln ($ Female Blood THg $)+0.6525$ & Egg THg=1.9203×Female Blood THg ${ }^{1.3734}$ \\
\hline Coraciiformes & 24 & 0.99 & 0.87 & $0.8602 \pm 0.0945$ & $-2.4879 \pm 0.1395$ & 39 & $\ln ($ Egg THg $)=0.8602 \times \ln ($ Female Blood THg) -2.4879 & Egg THg $=0.0831 \times$ Female Blood THg ${ }^{0.8002}$ \\
\hline Belted kingfisher & 24 & 0.99 & 0.87 & $0.8602 \pm 0.0945$ & $-2.4879 \pm 0.1395$ & 16 & $\ln ($ Egg THg $)=0.8602 \times \ln ($ Female Blood THg $)-2.4879$ & Egg THg $=0.0831 \times$ Female Blood THg ${ }^{0.8002}$ \\
\hline Gaviiformes & 125 & 0.94 & 0.54 & $0.7312 \pm 0.0858$ & $-0.6307 \pm 0.0946$ & 40 & $\ln (\mathrm{Egg} \mathrm{THg})=0.7312 \times \ln ($ Female Blood THg) -0.6307 & Egg THg $=0.5322 \times$ Female Blood THg ${ }^{0.7312}$ \\
\hline Common loon & 119 & 0.95 & 0.47 & $0.7218 \pm 0.0879$ & $-0.6271 \pm 0.1115$ & 22 & $\ln ($ Egg THg $)=0.7218 \times \ln ($ Female Blood THg $)-0.6271$ & Egg THg $=0.5341 \times$ Female Blood THg ${ }^{0.7218}$ \\
\hline Kenow et al. 2015; Wisconsin & 29 & 0.91 & 0.91 & $0.9753 \pm 0.0546$ & $-0.4318 \pm 0.0296$ & 6 & $\ln ($ Egg THg $)=0.9753 \times \ln ($ Female Blood THg $)-0.4318$ & Egg THg $=0.6493 \times$ Female Blood THg ${ }^{0.9753}$ \\
\hline Evers; northeastern USA & 90 & 0.94 & 0.42 & $0.7116 \pm 0.0931$ & $-0.6937 \pm 0.0671$ & 7 & $\ln ($ Egg THg $)=0.7116 \times \ln ($ Female Blood THg $)-0.6937$ & Egg THg $=0.4997 \times$ Female Blood THg ${ }^{0.7116}$ \\
\hline Yellow-billed loon & 6 & 0.50 & 0.50 & $0.6472 \pm 0.2889$ & $-0.7751 \pm 0.3878$ & 35 & $\ln ($ Egg THg $)=0.6472 \times \ln ($ Female Blood THg $)-0.7751$ & Egg THg $=0.4607 \times$ Female Blood $\mathrm{THg}^{0.6772}$ \\
\hline Passeriformes & 1050 & 0.98 & 0.71 & $0.8560 \pm 0.0198$ & $-1.4942 \pm 0.1911$ & 41 & $\ln (\mathrm{Egg} \mathrm{THg})=0.8560 \times \ln ($ Female Blood THg) -1.4942 & Egg THg $=0.2244 \times$ Female Blood THg ${ }^{0.8560}$ \\
\hline Carolina wren & 9 & 0.90 & 0.90 & $0.7090 \pm 0.0867$ & $-1.9443 \pm 0.0104$ & 19 & $\ln ($ Egg THg $)=0.7090 \times \ln ($ Female Blood THg $)-1.9443$ & Egg THg $=0.1431 \times$ Female Blood $\mathrm{THg}^{0.7000}$ \\
\hline Cristol; South River, Shenandoah Valley, Virginia & 4 & 0.99 & 0.99 & $0.7736 \pm 0.0183$ & $-1.9399 \pm 0.0325$ & 11 & $\ln ($ Egg THg $)=0.7736 \times \ln ($ Female Blood THg $)-1.9399$ & Egg THg $=0.1437 \times$ Female Blood THg ${ }^{0.7736}$ \\
\hline Evers; North Fork Holston River, Virginia & 5 & 0.54 & 0.54 & $0.4801 \pm 0.1524$ & $-1.9153 \pm 0.0949$ & 12 & $\ln ($ Egg THg $)=0.4801 \times \ln ($ Female Blood THg $)-1.9153$ & Egg THg $=0.1473 \times$ Female Blood THg ${ }^{0.4801}$ \\
\hline Eastern bluebird & 5 & 0.71 & 0.71 & $1.9391 \pm 0.9690$ & $-1.2038 \pm 0.1510$ & 24 & $\ln ($ Egg THg $)=1.9391 \times \ln ($ Female Blood THg $)-1.2038$ & Egg THg $=0.3001 \times$ Female Blood THg ${ }^{1.9391}$ \\
\hline Song sparrow & 25 & 0.96 & 0.87 & $1.0289 \pm 0.1293$ & $-1.2475 \pm 0.2198$ & 32 & $\ln (\mathrm{Egg} \mathrm{THg})=1.0289 \times \ln ($ Female Blood THg) -1.2475 & Egg THg $=0.2872 \times$ Female Blood THg ${ }^{1.028}$ \\
\hline Tree swallow & 568 & 0.98 & 0.87 & $0.8327 \pm 0.0225$ & $-1.2819 \pm 0.0887$ & 33 & $\ln ($ Egg THg $)=0.8327 \times \ln ($ Female Blood THg $)-1.2819$ & Egg THg $=0.2775 \times$ Female Blood THg ${ }^{0.8327}$ \\
\hline Ackerman et al. 2017; females bled during early incubation & 171 & 0.96 & 0.80 & $0.8091 \pm 0.0619$ & $-1.3740 \pm 0.0628$ & 1 & $\ln ($ Egg THg $)=0.8091 \times \ln ($ Female Blood THg $)-1.3740$ & Egg THg $=0.2531 \times$ Female Blood THg ${ }^{0.8091}$ \\
\hline Ackerman et al. 2017; females bled during late incubation & 52 & 0.70 & 0.70 & $0.7892 \pm 0.0718$ & $-1.2195 \pm 0.0490$ & 2 & $\ln (\mathrm{Egg} \mathrm{THg})=0.7892 \times \ln ($ Female Blood THg) -1.2195 & Egg THg $=0.2954 \times$ Female Blood THg ${ }^{0.7892}$ \\
\hline Brasso et al. 2010; females bled at clutch completion & 162 & 0.99 & 0.97 & $0.9598 \pm 0.0260$ & $-1.3412 \pm 0.0375$ & 3 & $\ln ($ Egg THg $)=0.9598 \times \ln ($ Female Blood THg $)-1.3412$ & Egg THg $=0.2615 \times$ Female Blood THg ${ }^{0.9598}$ \\
\hline Cristol; females bled throughout incubation & 137 & 0.99 & 0.97 & $0.8568 \pm 0.0243$ & $-0.9689 \pm 0.0354$ & 4 & $\ln ($ Egg THg $)=0.8568 \times \ln ($ Female Blood THg $)-0.9689$ & Egg THg $=0.3795 \times$ Female Blood THg ${ }^{0.8568}$ \\
\hline Evers; females bled throughout incubation & 46 & 0.92 & 0.63 & $0.4549 \pm 0.0728$ & $-1.8412 \pm 0.0984$ & 5 & $\ln ($ Egg THg $)=0.4549 \times \ln ($ Female Blood THg) -1.8412 & Egg THg $=0.1586 \times$ Female Blood THg ${ }^{0.449}$ \\
\hline Zebra finch & 104 & 0.92 & 0.64 & $0.7133 \pm 0.0954$ & $-0.2478 \pm 0.1944$ & 36 & $\ln ($ Egg THg $)=0.7133 \times \ln ($ Female Blood THg $)-0.2478$ & Egg THg $=0.7805 \times$ Female Blood THg ${ }^{0.7133}$ \\
\hline Procellariiformes & 3 & 0.17 & 0.17 & $0.3227 \pm 0.5010$ & $-1.3870 \pm 0.2002$ & 31 & $\ln ($ Egg THg $)=0.3227 \times \ln ($ Female Blood THg $)-1.3870$ & Egg THg $=0.2498 \times$ Female Blood THg ${ }^{0.3227}$ \\
\hline Northern fulmar & 3 & 0.17 & 0.17 & $0.3227 \pm 0.5010$ & $-1.3870 \pm 0.2002$ & 31 & $\ln ($ Egg THg $)=0.3227 \times \ln ($ Female Blood THg $)-1.3870$ & Egg THg $=0.2498 \times$ Female Blood THg ${ }^{0.3227}$ \\
\hline
\end{tabular}


Table S3. Equations (both on the natural log scale and back-transformed), model fit, and variance for predicting maternal blood total mercury concentrations ( $\mathrm{THg} \mu \mathrm{g} / \mathrm{g} \mathrm{ww}$ ) based on geometric mean egg THg concentrations $(\mu \mathrm{g} / \mathrm{g}$ fww) within her clutch for all taxa, 6 taxonomic orders, and 22 species of birds. Because there were no other fixed-effects in the models, marginal $R_{m}^{2}$ values indicated the explanatory power of THg concentrations in eggs for predicting THg concentrations in female bird blood. Conditional $R_{c}^{2}$ values described the proportion of variance explained by both the fixed- and random-effects in the model. 


\begin{tabular}{|c|c|c|c|c|c|c|c|}
\hline Taxa & $\begin{array}{r}\text { Sample } \\
\text { Size of } \\
\text { Nests }\end{array}$ & $R_{c}^{2}$ & $R_{m}^{2}$ & $\begin{array}{r}\text { Slope } \pm \text { SE } \\
\text { (log scale) }\end{array}$ & $\begin{array}{r}\text { Intercept } \pm \text { SE } \\
(\log \text { scale }) \\
\end{array}$ & Equation (log scale) & Equation (back-transformed) \\
\hline All taxa & 564 & 0.93 & 0.65 & $0.9179 \pm 0.0221$ & $0.7244 \pm 0.1511$ & $\ln ($ Female Blood THg $)=0.9179 \times \ln ($ Egg THg $)+0.7244$ & Female Blood $\mathrm{THg}=2.0635 \times \mathrm{Egg} \mathrm{THg}^{0.9179}$ \\
\hline Anseriformes & 89 & 0.88 & 0.79 & $0.9066 \pm 0.0954$ & $-0.1203 \pm 0.2086$ & $\ln ($ Female Blood THg $)=0.9066 \times \ln ($ Egg THg $)-0.1203$ & Female Blood THg $=0.8867 \times$ Egg THg ${ }^{0.9066}$ \\
\hline Common eider & 18 & 0.64 & 0.64 & $0.7677 \pm 0.1393$ & $-1.0308 \pm 0.2601$ & $\ln ($ Female Blood THg $)=0.7677 \times \ln ($ Egg THg $)-1.0308$ & Female Blood THg $=0.3567 \times$ Egg THg ${ }^{0.7677}$ \\
\hline Common goldeneye & 3 & 0.03 & 0.03 & $0.1463 \pm 0.5732$ & $-1.2471 \pm 0.9052$ & $\ln ($ Female Blood THg $)=0.1463 \times \ln ($ Egg THg $)-1.2471$ & Female Blood THg $=0.2873 \times$ Egg THg ${ }^{0.1463}$ \\
\hline Common merganser & 4 & 0.27 & 0.27 & $0.6379 \pm 0.6013$ & $0.3613 \pm 0.2801$ & $\ln ($ Female Blood THg $)=0.6379 \times \ln ($ Egg THg $)+0.3613$ & Female Blood THg=1.4352×Egg THg ${ }^{0.6379}$ \\
\hline Hooded merganser & 17 & 0.55 & 0.55 & $1.1063 \pm 0.2505$ & $0.0912 \pm 0.2258$ & $\ln ($ Female Blood THg $)=1.1063 \times \ln ($ Egg THg $)+0.0912$ & Female Blood THg $=1.0955 \times E_{\text {Eg THg }}{ }^{1.1063}$ \\
\hline Mallard & 39 & 0.92 & 0.91 & $0.9971 \pm 0.0702$ & $0.0709 \pm 0.1093$ & $\ln ($ Female Blood THg $)=0.9971 \times \ln (\mathrm{Egg} \mathrm{THg})+0.0709$ & Female Blood $\mathrm{THg}=1.0735 \times \mathrm{Egg} \mathrm{THg}^{0.9971}$ \\
\hline Wood duck & 8 & 0.13 & 0.13 & $0.2457 \pm 0.2397$ & $-2.4231 \pm 0.8144$ & $\ln ($ Female Blood THg $)=0.2457 \times \ln ($ Egg THg $)-2.4231$ & Female Blood THg $=0.0886 \times$ Egg THg ${ }^{0.2457}$ \\
\hline Charadriiformes & 100 & 0.90 & 0.65 & $0.7926 \pm 0.0537$ & $0.5148 \pm 0.3950$ & $\ln ($ Female Blood THg $)=0.7926 \times \ln (\operatorname{Egg~THg})+0.5148$ & Female Blood THg $=1.6733 \times$ Egg THg ${ }^{0.7926}$ \\
\hline American avocet & 25 & 0.76 & 0.76 & $0.8272 \pm 0.0887$ & $0.6285 \pm 0.1455$ & $\ln ($ Female Blood THg $)=0.8272 \times \ln ($ Egg THg $)-0.6285$ & Female Blood THg=1.8748 $\times \operatorname{Egg~THg}^{0.8272}$ \\
\hline Black-legged kittiwake & 15 & 0.98 & 0.07 & $0.6143 \pm 0.1167$ & $0.0928 \pm 0.7918$ & $\ln ($ Female Blood THg $)=0.6143 \times \ln ($ Egg THg $)+0.0928$ & Female Blood THg=1.0972×Egg THg ${ }^{0.6143}$ \\
\hline Black-necked stilt & 29 & 0.77 & 0.77 & $1.0808 \pm 0.1101$ & $0.7526 \pm 0.1017$ & $\ln ($ Female Blood THg $)=1.0808 \times \ln ($ Egg THg $)+0.7526$ & Female Blood THg $=2.1225 \times$ Egg THg ${ }^{1.0808}$ \\
\hline Forster's tern & 17 & 0.30 & 0.30 & $0.4031 \pm 0.1451$ & $0.7088 \pm 0.0685$ & $\ln ($ Female Blood THg $)=0.4031 \times \ln (\mathrm{Egg} \mathrm{THg})+0.7088$ & Female Blood $\mathrm{THg}=2.0316 \times \mathrm{Egg} \mathrm{THg}^{0.4031}$ \\
\hline Herring gull & 12 & 0.67 & 0.67 & $0.5018 \pm 0.1066$ & $-1.0105 \pm 0.2612$ & $\ln ($ Female Blood THg $)=0.5018 \times \ln ($ Egg THg $)-1.0105$ & Female Blood $\mathrm{THg}=0.3640 \times \operatorname{Egg} \mathrm{THg}^{0.5018}$ \\
\hline Coraciiformes & 7 & 0.93 & 0.93 & $1.0954 \pm 0.1207$ & $2.7083 \pm 0.3638$ & $\ln ($ Female Blood THg $)=1.0954 \times \ln ($ Egg THg $)+2.7083$ & Female Blood THg=15.0037×Egg THg ${ }^{1.0954}$ \\
\hline Belted kingfisher & 7 & 0.93 & 0.93 & $1.0954 \pm 0.1207$ & $2.7083 \pm 0.3638$ & $\ln ($ Female Blood THg $)=1.0954 \times \ln (\mathrm{Egg} \mathrm{THg})+2.7083$ & Female Blood THg=15.0037×Egg THg ${ }^{1.0954}$ \\
\hline Gaviiformes & 98 & 0.67 & 0.35 & $0.6560 \pm 0.0711$ & $0.1921 \pm 0.3549$ & $\ln ($ Female Blood THg $)=0.6560 \times \ln ($ Egg THg $)+0.1921$ & Female Blood THg=1.2118×Egg THg ${ }^{0.6560}$ \\
\hline Common loon & 93 & 0.58 & 0.38 & $0.6436 \pm 0.0712$ & $0.4293 \pm 0.2268$ & $\ln ($ Female Blood THg $)=0.6436 \times \ln ($ Egg THg $)+0.4293$ & Female Blood THg $=1.5362 \times$ Egg THg ${ }^{0.6436}$ \\
\hline Yellow-billed loon & 5 & 0.73 & 0.73 & $1.1209 \pm 0.3405$ & $0.5506 \pm 0.5448$ & $\ln ($ Female Blood THg $)=1.1209 \times \ln ($ Egg THg $)+0.5506$ & Female Blood THg=1.7343×Egg THg ${ }^{1.1209}$ \\
\hline Passeriformes & 267 & 0.96 & 0.81 & $1.0221 \pm 0.0246$ & $1.4625 \pm 0.1937$ & $\ln ($ Female Blood THg $)=1.0221 \times \ln ($ Egg THg $)+1.4625$ & Female Blood THg $=4.3167 \times$ Egg THg ${ }^{1.0221}$ \\
\hline Carolina wren & 6 & 0.96 & 0.96 & $1.3426 \pm 0.1372$ & $2.6735 \pm 0.2354$ & $\ln ($ Female Blood THg $)=1.3426 \times \ln (\mathrm{Egg} \mathrm{THg})+2.6735$ & Female Blood THg=14.4906 $\times \operatorname{Egg~THg}^{1.3426}$ \\
\hline Eastern bluebird & 3 & 0.67 & 0.67 & $0.4128 \pm 0.2062$ & $0.4871 \pm 0.2758$ & $\ln ($ Female Blood THg $)=0.4128 \times \ln ($ Egg THg $)+0.4871$ & Female Blood THg=1.6276×Egg THg ${ }^{0.4128}$ \\
\hline House wren & 45 & 0.87 & 0.87 & $0.8319 \pm 0.0488$ & $1.3481 \pm 0.0995$ & $\ln ($ Female Blood THg $)=0.8319 \times \ln ($ Egg THg $)+1.3481$ & Female Blood THg=3.8501×Egg THg ${ }^{0.8319}$ \\
\hline Indigo bunting & 10 & 0.82 & 0.82 & $1.0484 \pm 0.1646$ & $2.3146 \pm 0.5422$ & $\ln ($ Female Blood THg $)=1.0484 \times \ln ($ Egg THg $)+2.3146$ & Female Blood THg $=10.1209 \times$ Egg THg ${ }^{1.0484}$ \\
\hline Song sparrow & 7 & 0.92 & 0.92 & $0.8976 \pm 0.1101$ & $1.0680 \pm 0.3139$ & $\ln ($ Female Blood THg $)=0.8976 \times \ln ($ Egg THg $)+1.0680$ & Female Blood THg=2.9096×Egg THg ${ }^{0.8976}$ \\
\hline Tree swallow & 165 & 0.90 & 0.87 & $1.0701 \pm 0.0303$ & $1.2858 \pm 0.1002$ & $\ln ($ Female Blood THg $)=1.0701 \times \ln ($ Egg THg $)+1.2858$ & Female Blood THg=3.6176×Egg THg ${ }^{1.0701}$ \\
\hline Zebra finch & 28 & 0.69 & 0.69 & $0.9461 \pm 0.1234$ & $0.8833 \pm 0.1521$ & $\ln ($ Female Blood THg $)=0.9461 \times \ln ($ Egg THg $)+0.8833$ & Female Blood THg=2.4189 $\times$ Egg THg $^{0.9461}$ \\
\hline Procellariiformes & 3 & 0.17 & 0.17 & $0.9088 \pm 1.4107$ & $1.0797 \pm 2.0891$ & $\ln ($ Female Blood THg $)=0.9088 \times \ln ($ Egg THg $)+1.0797$ & Female Blood $\mathrm{THg}=2.9438 \times \mathrm{Egg} \mathrm{THg}^{0.9088}$ \\
\hline Northern fulmar & 3 & 0.17 & 0.17 & $0.9088 \pm 1.4107$ & $1.0797 \pm 2.0891$ & $\ln ($ Female Blood THg $)=0.9088 \times \ln ($ Egg THg $)+1.0797$ & Female Blood THg $=2.9438 \times$ Egg THg $^{0.9088}$ \\
\hline
\end{tabular}

